\title{
Non-Surgical Management of Iatrogenic Perforation in Mandibular Molar Using Mineral Trioxide Aggregate Followed by Composite Onlay: A Case Report
}

\author{
Henytaria Fajrianti ${ }^{1}$ Adi Nugroho H.K. ${ }^{2}$ Yulita Kristanti ${ }^{3}$ Diatri Nari Ratih ${ }^{4, *}$ \\ ${ }^{1,2}$ Conservative Dentistry Specialty Program, Faculty of Dentistry, Universitas Gadjah Mada \\ ${ }^{3,4}$ Department of Conservative Dentistry, Faculty of Dentistry, Universitas Gadjah Mada \\ *Diatri Nari Ratih. Email: diatri@ugm.ac.id
}

\begin{abstract}
One of the most common iatrogenic during endodontic is a perforation, especially furcation perforation on the molar. Perforation creates an artificial communication between a root canal and periodontal tissue, which could lead to pulpal inflammation extends to the periodontium. Management of perforation is a challenging task for clinicians' inconsideration of poor accessibility and visibility. This case report aims to present a case of furcal perforation treatment by a non-surgical approach. A 24 -year-old patient was referred to the Conservative Dental Clinic Prof. Soedomo Dental Hospital to continue root canal treatment on a mandibular first molar (36). The patient had been treated by two dentists before. Clinical examination showed a black area on the floor of the pulp chamber. The radiographic examination showed a little radiolucent area in the furcal region of tooth 36 . The palpation test did not reveal any tenderness with no mobility but had percussion pain. An artificial wall was built on the distal wall. Root canal treatment was done by a rotary file with the crown down technique on three canals. Furcation perforation was treated under endodontic microscopic. Mineral trioxide aggregate (MTA) was placed on the perforation by using the MTA carrier. After MTA has set, bulk-fill and fiber-reinforced composite were placed on building up the cavity. Final restoration made by direct-indirect technique with onlay composite, Conclusion, non-surgical approach of perforation treatment could be done by using MTA under good magnification to achieve proper material placement on the perforation. MTA has a good sealability to repair perforation, and this case could be done successfully.
\end{abstract}

Keywords: Iatrogenic, furcal perforation, MTA, root canal treatment

\section{INTRODUCTION}

Root canal treatment is mainly aimed to obtain the hermetic obturation on the root canal to avoid re-infection. The re-infection of the root canal could be caused by inadequacies during the shaping, cleaning, and obturation process (1). One of the undesired complications that can occur during the preparation of endodontic access cavities or exploring the canal orifice of multirooted teeth is perforation. Perforation on the furcation floor is the most common iatrogenic complication that can harm root canal treatment prognosis (2). This situation could be managed surgically or non-surgically (3).

Both on surgical and non-surgically, ideal biomaterial needs to seal the perforation. The material should be antibacterial, biocompatible, non-toxic, non-absorbable, radiopaque, and have the ability to induce the formation of hard tissue (4). A wide variety of materials are used to repair the perforation, such as amalgam, zinc-oxide eugenol (ZOE), glass ionomer, resin-modified glass ionomer, and calcium hydroxide (5).
Recently, Mineral Trioxide Aggregate (MTA) has been proposed as an alternative to sealing perforation. MTA consists of fine hydrophilic particles of tricalcium silicate, silicate oxide, and tricalcium oxide. The setting time of MTA is 3-4 hours, with biocompatibility and bacteriostatic action (6). Therefore, MTA has been indicated as a suitable material for only a single visit perforation treatment.

This article aims to report a case of furcation perforation due to iatrogenic by non-surgical approach. Furcation perforation seal with MTA, which could induce cementogenesis and osteogenesis on the perforation site.

\section{CASE REPORT}

A 24-year-old female patient was referred to conservative clinic Prof Soedomo Dental Hospital by a general dentist to continue root canal treatment of the left mandibular molar. Subjective examination revealed there was spontaneous pain on the left mandibular tooth. Patient under orthodontic treatment for one year. Her left mandibular molar had been treated by two general dentists before. Clinical examination showed a disto-occlusal cavity on 36 , which had reached the 
pulp chamber, and a dark spot on the distolingual pulp floor, which was suspected as perforation (Figure 1).

The palpation and percussion test did not reveal any tenderness with no mobility, and periodontal probing around the tooth was within physiological limits. Besides, medical history did not make any contribution. Also, no significant family history was revealed, and extraoral findings were unremarkable. Radiographic examination revealed a small radiolucent area in the furcal region of 36 (Figure 2). Tooth \#36 was diagnosed as pulp necrose with symptomatic apical periodontitis.

Therefore, the treatment plan was perforation sealing with mineral trioxide aggregate (non-surgical) followed by composite onlay. Informed consent was obtained from the patient. All treatment procedures were done under rubber dam isolation. The temporary restoration was removed, and an artificial wall was built by using composite the disto-occlusal site (Figure 3). The root canals were explored using \#10 Kfile (Dentsply, Switzerland). Coronal flaring was done by using the SX file (Protaper Gold, Dentsply, Switzerland). The working length was determined by an electronic apex locator (Propex Pixi, Dentsply, Switzerland) and confirmed by the radiograph, which was $19 \mathrm{~mm}$ on mesiobuccal, $17 \mathrm{~mm}$ on mesiolingual, and $19 \mathrm{~mm}$ on distal root canal (Figure 4). Proglider file (Dentsply, Switzerland) was used to create a glide path. Root canals were prepared by using Reciproc Blue File \#25 (VDW, Munich). Sodium hypochlorite $(\mathrm{NaOCl})$ with a concentration of $2.5 \%$ was used as an irrigant solution during instrumentation, and apical gauging was reached by using K-file \#25. Absorbent points were used to dry the root canal, and mixture paste of $\mathrm{Ca}(\mathrm{OH}) 2$ with glycerin was placed into the root canal as an intracanal medicament and sealed with a temporary filling. The patient was then called again after a week.

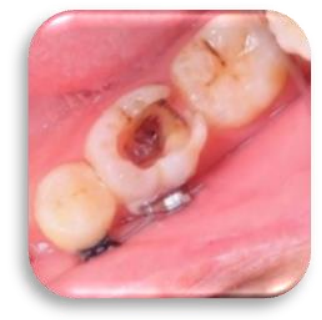

Figure 1 Preoperative Clinical Examination

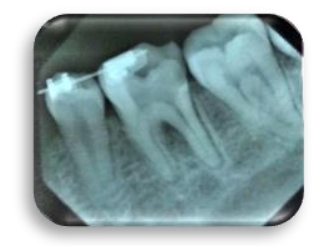

Figure 2 Preoperative intraoral periapical radiograph

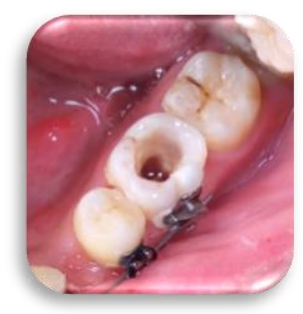

Figure 3 Artificial wall on disto-occlusal

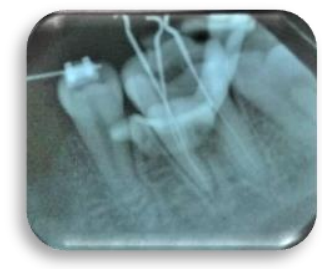

Figure 4 Working length confirmed by radiograph

On the second visit, the tooth was asymptomatic, and the clinical examination revealed no swelling on the gingival. Meanwhile, the percussion test showed a negative result. Intracanal medicament was cleaned using $2.5 \% \mathrm{NaOCl}$ as the irrigant. The trial of the master apical cone was confirmed with a periapical radiograph (Figure 5). The tooth was irrigated using $2.5 \% \mathrm{NaOCl}, 17 \%$ EDTA, and $2 \%$ Chlorhexidine. Distilled water was employed after applying each irrigant solution. The root canal was dried by a sterile absorbent point. Obturation was done by using epoxy resin (AH Plus, Dentsply) followed with a continuous-wave compaction method and was evaluated by a periapical radiograph (Figure 6).

The next phase of the treatment was the removal of temporary filling. The percussion and palpation test showed a negative result. Treatment was done under an endodontic microscope (Zumax). The perforation site was cleaned and irrigated using $2.5 \% \mathrm{NaOCl}, 17 \%$ EDTA, and $2 \%$ Chlorhexidine (Figure 7). Distilled water was employed after applying each irrigant solution. Mineral trioxide aggregate (MTA) (Pro-Root, Dentsply, Switzerland) was mixed with distilled water as the manufacturer's instructions into a thick paste and placed in the apical third using a plugger until the perforation site was sealed fully (Figure 8). A cotton pellet was then placed in the pulp chamber to produce a humid ambient for the MTA to achieve its solidification, and the tooth was temporarily filled with temporary restoration material. A periapical radiograph was taken to evaluate the treatment (Figure 9)

At the next meeting, the tooth was asymptomatic, and the clinical examination revealed no swelling on the gingival. Meanwhile, the percussion test showed a negative result. Bulk-fill and fiber-reinforced composite were placed on the base of the cavity, and the tooth was prepared to made onlay restoration (Figure 10 and Figure 11). Rubber base impression was taken. Onlay restoration was done on silicone mold with the semi-direct method (Figure 12) 
The next treatment phase was onlay insertion. Onlay restoration was done with $37 \%$ etch and silane on the fitting surface area. The cavity was cleaned with a rotary brush and pumice. Onlay restoration was cemented, and a periapical radiograph was taken (Figure 13).

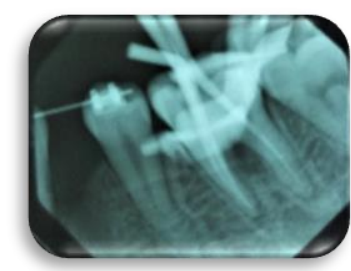

Figure 5 Master Apical Cone trial

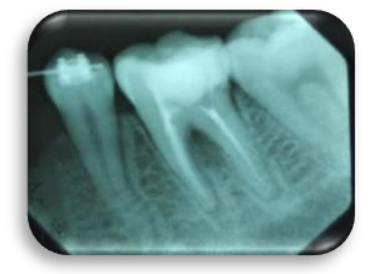

Figure 6 Post obturation radiograph

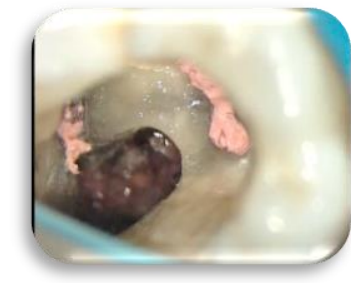

Figure 7 Perforation site had been cleaned and was treated under an endodontic microscope

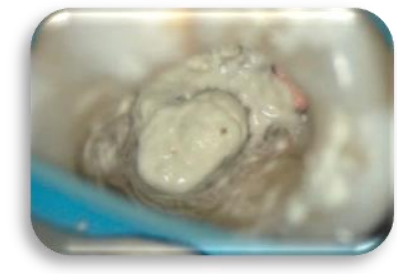

Figure 8 Perforation site was sealed with MTA

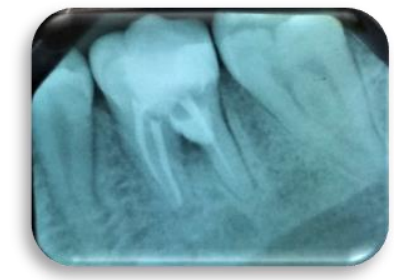

Figure 9 Sealing of perforation site was evaluated with radiograph

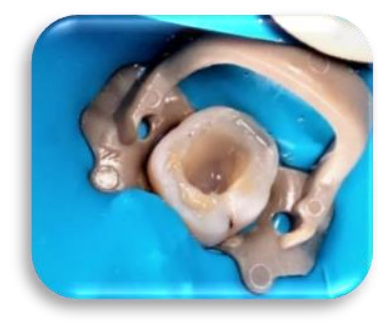

Figure 10 Bulk-fill and fiber-reinforced composite was placed on the base of the cavity

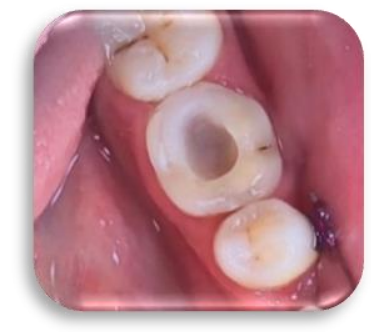

Figure 11 Onlay preparation

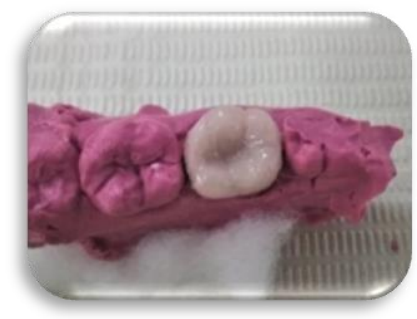

Figure 12 Composite onlay was done with semi-direct technique on the silicone mould
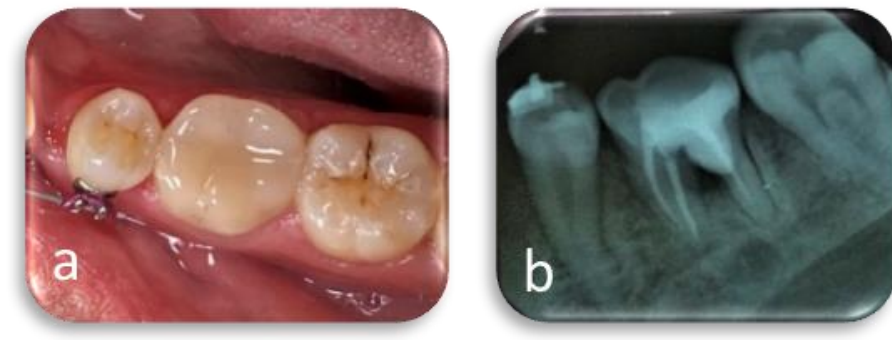

Figure 13 (a) Cementation of composite onlay, (b) post cementation radiograph

\section{DISCUSSION}

Furcal perforation might occur during root canal treatment due to caries or iatrogenic. Lack of anatomical knowledge and poor magnification is the most reason for this iatrogenic event. Perforations during endodontic procedures are cited as the second greatest cause of treatment failures (2).

Many factors can affect the prognosis of furcation perforation, such as the location and level of the lesion and size of perforation. One of the most important considerations in perforation repair is the time elapsed between the accident and its repair. The success rate increases with a decrease in time interval on this event (7). The location of furcation and the level of perforation in the relation of the crestal bone are challenging. Good magnification has to be achieved on the non-surgical approached to repair the furcation perforation. 
A tightly sealed in perforation site is the key to successful treatment. It aimed to eliminated microbial contamination and guarded the periodontium tissue to achieve optimal healing (3). In the past years, amalgam, composite resin, and glass ionomer cement have been used for sealing furcal perforation (4). Another material that had been used to repair perforation, such as tin foil, paraffin, gutta-percha, gold foil, ZnPO4 cement, formulations of $\mathrm{Ca}(\mathrm{OH}) 2$, iodoform, $\mathrm{ZnOE}$, cavity, dentin chips, indium foil, EBA cement, and gel foam (5). Recently, Mineral Trioxide Aggregate (MTA) has been developed for perforation treatment. The MTA has superior sealing property, biocompatibility, ability to set in the presence of blood, and can induce cementum-like hard tissue (8). Moreover, MTA has less leakage, high marginal adaptation, better antibacterial properties, short setting time (4 hours), and a pH of 12,5. MTA stimulates the production of interleukins and cytokines to promote hard tissue formation (7). The desirable properties of MTA make this material could become alternative material which useful in repairing root and furcal perforation.

In this case, recovery was achieved by creating optimal conditions despite a time interval between perforation and repair treatment. The perforation area should be disinfected successfully. The contaminated perforation area could be washed out with hypochlorite. Good response in which repair with MTA can likely be attributed to the effective use of hypochlorite irrigation. Dry environment and good magnifications under the endodontic microscope create better visuals to be placed on the material on the perforation site and create a tight sealing on it (4).

Restoration on treated teeth should be considered due to stress and occlusal force on it, particularly on the posterior tooth. Replacement of large size restorations using composite resin has raised some concerns, including polymerization shrinkage stress that may cause poor marginal adaptation and recurrent carries (9). Therefore, bulk-filling composite resin (SDR, Dentsply) was chosen to replace the lost dentin structure in this present case to reduce the polymerization shrinkage and to improve the tooth-restoration interface adaptation before final restoration (10).

Due to the shrinkage of composite, semi-direct restoration could be an alternative to a large cavity. The indirect technique in particular allows the initial polymerization contraction and the following post-curing shrinkage to occur before cementation (11). Marginal adaptation of resin composite onlay has been reported in some studies to be superior to direct fillings, showing less microleakage and better marginal quality. Also, tensile strength, elastic modulus, fracture toughness, hardness, and wear resistance are improved by post-curing (12).

Semi-direct onlay could be done with a free-hand semidirect technique. It does not need expensive special equipment. The semi-direct technique acquires the advantages of both direct and indirect techniques. This technique eliminates lab involvement and could be done in one meeting (9). The materials used should be indifferent chemical composition with the intention of ensuring east separation between the impression and the model. Silicon die was introduced to have a certain precision in the working model. Semi-direct composite resin inlay/onlay lasts longer with an improved marginal adaptation and a low incidence of secondary caries (12).

\section{CONCLUSIONS}

Complications due to iatrogenic perforation may occur during root canal treatment. A tight seal could be achieved by MTA as a potential material for repair furcal perforation. Good magnification and disinfection become determining factors that lead to a successful non-surgical perforation repair. Semi-direct onlay restoration could become an alternative final restoration on the treated tooth, which has advantages such as less cost, less time, and less equipment.

\section{REFERENCES}

(1) Ramaprabha Balasubramaniam, D., Krishnan, A., \& Jayakumar, S. (2017). Restoring the dignity: Case reports of root perforation management.

(2) Unal, G. C., Maden, M., \& Isidan, T. (2010). Repair of furcal iatrogenic perforation with mineral trioxide aggregate: two years follow-up of two cases. European journal of dentistry, 4(4), 475.

(3) do Carmo Monteiro, J. C., Tonetto, M. R., Bandeca, M. C., Borges, A. H., Segalla, J. C. M., Jordão-Basso, K. C. F., ... \& Kuga, M. C. (2017). Repair of iatrogenic furcal perforation with mineral trioxide aggregate: A sevenyear follow-up. Iranian Endodontic Journal, 12(4), 516.

(4) Mohammadzadeh-Akhlagi, N., \& Esnaashari, E. (2015). Repair of a furcation perforation with mineral trioxide aggregate: A case report with 6 year follow-ups. Journal of Oral Health and Oral Epidemiology, 4(2), 102-106.

(5) de Sousa Reis, M., Scarparo, R. K., Steier, L., \& de Figueiredo, J. A. P. (2019). Periradicular inflammatory response, bone resorption, and cementum repair after sealing of furcation perforation with mineral trioxide aggregate (MTA Angelus ${ }^{\mathrm{TM}}$ ) or biodentine ${ }^{\mathrm{TM}}$. Clinical oral investigations, 23(11), 4019-4027.

(6) Majeed, H. A., Tirmazi, S. M., \& Iqbal, N. (2017). Furcal perforation repair: MTA versus calcium hydroxide. Pakistan Oral \& Dental Journal, 37(2), 317-320.

(7) Hosoya, N., Takigawa, T., Horie, T., Maeda, H., Yamamoto, Y., Momoi, Y., \& Okiji, T. (2019). A review of the literature on the efficacy of mineral trioxide aggregate in conservative dentistry. Dental materials journal, 38(5), 693-700.

(8) Haque F, Ahmed Z, Rime SA, Hossain MM, Chowdhury AA, Khan M. An Overview of Mineral Trioxide Aggregate in Apexification. Journal of Advances in Medicine and Medical Research. 2017 Jul 17:1-7. 
(9) Alharbi, A., Rocca, G. T., Dietschi, D., \& Krejci, I. (2014). Semidirect composite onlay with cavity sealing: a review of clinical procedures. Journal of esthetic and restorative dentistry, 26(2), 97-106.

(10) Sufyan Garoushi, B. D. S., Shinya, A., Shinya, A., \& Vallittu, P. K. (2009). Fiber-reinforced onlay composite resin restoration: a case report. The Journal of Contemporary Dental Practice, 10(4).
(11) Alharbi, A., Rocca, G. T., Dietschi, D., \& Krejci, I. (2014). Semidirect composite onlay with cavity sealing: a review of clinical procedures. Journal of esthetic and restorative dentistry, 26(2), 97-106.

(12) Asyraf, B. M., \& Lim, T. W. (2016). Semi-Direct Composite Restorations-A Clinical Report. Malaysian Dental Journal, 2. 